\title{
The complete chloroplast genome of Limonium tetragonum (Plumbaginaceae) isolated in Korea
}

\author{
Yongsung KIM, Hong $\mathrm{XI}^{\mathbf{1}}$ and Jongsun PARK ${ }^{1 *}$ \\ Honam National Institute of Biological Resources, Mokpo 58762, Korea \\ ${ }^{I}$ InfoBoss Inc. and InfoBoss Research Center, Seoul 06278, Korea \\ (Received 27 June 2021; Revised 16 August 2021; Accepted 7 September 2021)
}

\begin{abstract}
The chloroplast genome of Limonium tetragonum (Thunb.) Bullock, a halophytic species, was sequenced to understand genetic differences based on its geographical distribution. The cp genome of L. tetragonum was 154,689 bp long (GC ratio is $37.0 \%$ ) and has four subregions: 84,572 bp of large single-copy (35.3\%) and 12,813 bp of small singlecopy (31.5\%) regions were separated by 28,562 bp of inverted repeat ( $40.9 \%)$ regions. It contained 128 genes ( 83 proteincoding genes, eight rRNAs, and 37 tRNAs). Thirty-five single-nucleotide polymorphisms and 33 INDEL regions ( $88 \mathrm{bp}$ in length) were identified. Maximum-likelihood and Bayesian inference phylogenetic trees showed that L. tetragonum formed a sister group with L. aureum, which is incongruent with certain previous studies, including a phylogenetic analysis.
\end{abstract}

Keywords: Limonium tetragonum, chloroplast genome, phylogenetic analysis, intraspecific variation, Plumbaginaceae

Limonium, belonging to Plumbaginaceae family, is a cosmopolitan halophytic genus, and a few species inhabit alkaline soil away from the coastal area (Kubitzki, 1993; Morgan and Funnell, 2018). Limonium tetragonum (Thunb.) Bullock, distributed in coastal areas of Korea and Japan, is a biennial halophytic species with radical leaves $(8-15 \times 1.5-3 \mathrm{~cm})$ and yellow corolla (Owhi, 1965; Park, 2007; Lee et al., 2011; Park et al., 2020a). The crude extracts and solvent-partitioned fractions of whole plants of $L$. tetragonum display antioxidant (Lee et al., 2011) and anti-cancer activities (Kong et al., 2008); ethyl acetate soluble fraction of aerial parts exhibits anti-liver fibrosis (Kim et al., 2016); that of whole plants shows anti-alcohol toxicity (Kim et al., 2015); and its methanol extracts of whole plants present hepatoprotective activities (Yang et al., 2014). We sequenced $L$. tetragonum from the western coastal area of Korea for investigating intraspecific variations on chloroplast genomes with chloroplast of $L$. tetragonum isolated in eastern seashore of Korea.

\section{Materials and Methods}

\section{Plant material}

We collected L. tetragonum in Aphaedo island in Shinan- gun, Jeollanam-do, Korea (34.8612N, 126.32629E). A voucher specimen and genomic DNA were deposited in the InfoBoss Cyber Herbarium (IN, the voucher number IB-00899).

\section{DNA extraction and chloroplast genome determination}

The total genomic DNA was extracted from fresh leaf by using a DNeasy Plant Mini Kit (QIAGEN, Hilden, Germany). Genome sequencing was performed using HiSeqX at Macrogen Inc., Korea, and de novo assembly was done by Velvet v1.2.10 (Zerbino and Birney, 2008) and GapCloser v1.12 (Zhao et al., 2011). Assembled sequences were confirmed by BWA v0.7.17 (Li, 2013) and SAMtools v1.9 (Li et al., 2009). All bioinformatic analyses were conducted in the Genome Information System (http://geis.infoboss.co.kr/) utilized in the previous studies (Kim et al., 2018, 2019a, 2019b, 2019c, 2021; Bum et al., 2020; Park et al., 2021c).

Genome annotation was conducted based on another $L$. tetragonum chloroplast (MW085088.1) with Geneious R11 v11.0.5 (Biomatters Ltd, Auckland, New Zealand). A circular map of L. tetragonum chloroplast genome was drawn using OGDRAW v1.31 (Greiner et al., 2019).

\footnotetext{
*Author for correspondence: starflr@infoboss.co.kr
} 


\section{Identification of intraspecific variations}

Single nucleotide polymorphisms (SNPs) and insertions and deletions (INDELs) were identified using the 'Find variations/ SNPs' function implemented in the Geneious R11 v11.0.5 (Biomatters Ltd, Auckland, New Zealand) based on the pairwise alignment of the two chloroplast genomes of L. tetragonum conducted by MAFFT 7.450 (Katoh and Standley, 2013). This method has been used in the previous studies (Kim et al., 2019a; Min et al., 2019a; Choi et al., 2021; Park et al., 2021b, 2021d). INDEL region was defined as the continuous INDELs.

\section{Phylogenetic analysis}

Maximum-likelihood (ML) and Bayesian inference (BI) phylogenetic trees were constructed based on the multiple sequence alignment of all available nine Plumbaginaceae chloroplast genomes and the seven chloroplast genomes of non-core Caryophyllales clade (Crawly and Hilu, 2012; Yao et al., 2019) by MAFFT v7.450 (Katoh and Standley, 2013). The chloroplast genome of Nepenthes graciliflora Elmer (1912) (Yao et al., 2019) was marked as outgroup species. The ML tree was reconstructed in IQ-TREE v1.6.12 (Nguyen et al., 2015) with 1,000 bootstrap repeats. In the ML analysis, a heuristic search was used with nearest-neighbor interchange branch swapping, GTR $+\mathrm{F}+\mathrm{R} 4$ model determined as the best fit model by the ModelFinder implemented in IQ-TREE, and uniform rates among sites. All other options used the default settings. The posterior probability of each node was estimated by the BI using MrBayes v3.2.6 (Huelsenbeck and Ronquist 2001). The HKY85 model with gamma rates was used as a molecular model. A Markov chain Monte Carlo algorithm was employed for 1,100,000 generations, sampling trees every 200 generations, with four chains running simultaneously. Trees from the first 100,000 generations were discarded as burn-in.

\section{Data availablity}

Chloroplast genome sequence can be accessed via accession number of MN044572 in GenBank of NCBI at https:// www.ncbi.nlm.nih.gov. The associated BioProject, SRA, and Bio-Sample numbers are PRJNA737051, SAMN19678692, and SRR14793527, respectively.

\section{Results and Discussion}

The chloroplast genome of $L$. tetragonum (GenBank accession of MN044572) isolated in Korea is 154,689 bp long (GC ratio of $37.0 \%$ ) and had four subregions: $84,572 \mathrm{bp}$ of large single copy (35.3\%) and 12,813 bp of small single copy (31.5\%) regions are separated by 28,562 bp of inverted repeat (IR; $40.9 \%$ ) (Table 1), which is similar to those of the previously reported L. tetragonum chloroplast genome (MW085088) (Table 1). It contained 128 genes (83 protein-coding genes [PCGs], eight rRNAs, and 37 tRNAs); 14 genes (five PCGs, four rRNAs, and five tRNAs) were duplicated in the IR regions, which is also same to those of the previously reported $L$. tetragonum chloroplast genome (MW085088) (Fig. 1, Table 1).

Based on the pair-wise sequence alignment with the previously reported L. tetragonum chloroplast genome (MW085088) (Darshetkar et al., 2021), 35 SNPs and 33 INDELs regions ( $88 \mathrm{bp}$ in total) were identified. The longest INDEL region was 18-bp located between trnT and trnL. The inserted sequence was 18 -bp repetitive sequences. In addition, two chloroplast genomes of Limonium bicolor (Bunge) Kuntze (Darshetkar et al., 2021) display extremely divergent manner, no SNP and 116 INDEL regions (15,840 bp in length), which is similar to the case of Phedimus takesimensis chloroplast genomes (129 SNPs and 112 INDEL regions (8,506 bp in length) (Park et al., in preparation).

Numbers of these intraspecific variations of $L$. tetragonum were smaller than those of Pseudostellaria palibiniana (Takeda) Ohwi (84 SNPs and 125-bp INDELs), Pyrus ussuriensis Maxim. (1,221 SNPs and 781-bp INDELs), Goodyera schlechtendaliana Rchb. f. (200 SNPs and 511-bp INDELs), Gastrodia elata Blume (324 SNPs and 630-bp INDELs) isolated in Korea; while they were larger than those of Artemisia fukudo Makino (seven SNPs and 12-bp INDELs) (Min et al., 2019b), Fagus multinervis Nakai (two SNPs and 2-bp INDELs) (Park and Oh, 2020), Aconitum coreanum (H. Lév.) Rapaics (five to 19 SNPs and 52-bp to 950bp INDELs) (Kim et al., 2019d), Viburnum erosum Thunb. (16 SNPs and 50-bp INDELs) (Choi et al., 2020), and Veronica nakaiana Ohwi (seven SNPs and 4-bp INDELs) (Lee et al., 2021). In

Table 1. List of two available chloroplast genomes of Limonium tetragonum.

\begin{tabular}{|c|c|c|c|c|c|c|c|c|c|c|c|}
\hline \multirow{2}{*}{$\begin{array}{l}\text { GenBank } \\
\text { accession }\end{array}$} & \multicolumn{4}{|c|}{ Length (bp) } & \multicolumn{4}{|c|}{ GC contents } & \multicolumn{3}{|c|}{ No. of genes } \\
\hline & Whole & LSC & $\mathrm{SSC}$ & IR & $\begin{array}{c}\text { Whole } \\
(\%)\end{array}$ & $\begin{array}{c}\text { LSC } \\
(\%)\end{array}$ & $\begin{array}{l}\text { SSC } \\
(\%)\end{array}$ & $\begin{array}{l}\text { IR } \\
(\%)\end{array}$ & $\begin{array}{l}\text { No. of } \\
\text { PCGs }\end{array}$ & $\begin{array}{l}\text { No. of } \\
\text { tRNAs }\end{array}$ & $\begin{array}{l}\text { No. of } \\
\text { rRNAs }\end{array}$ \\
\hline MN044572 & 154,689 & 84,572 & 13,013 & 28,562 & 37.0 & 35.3 & 31.5 & 40.9 & 83 & 37 & 8 \\
\hline MW085088 & 154,691 & 84,568 & 12,997 & 28,563 & 37.0 & 35.3 & 31.5 & 40.9 & 83 & 37 & 8 \\
\hline
\end{tabular}

LSC, large single copy; SSC, small single copy; IR, inverted repeat ; PCG, protein-coding gene. 


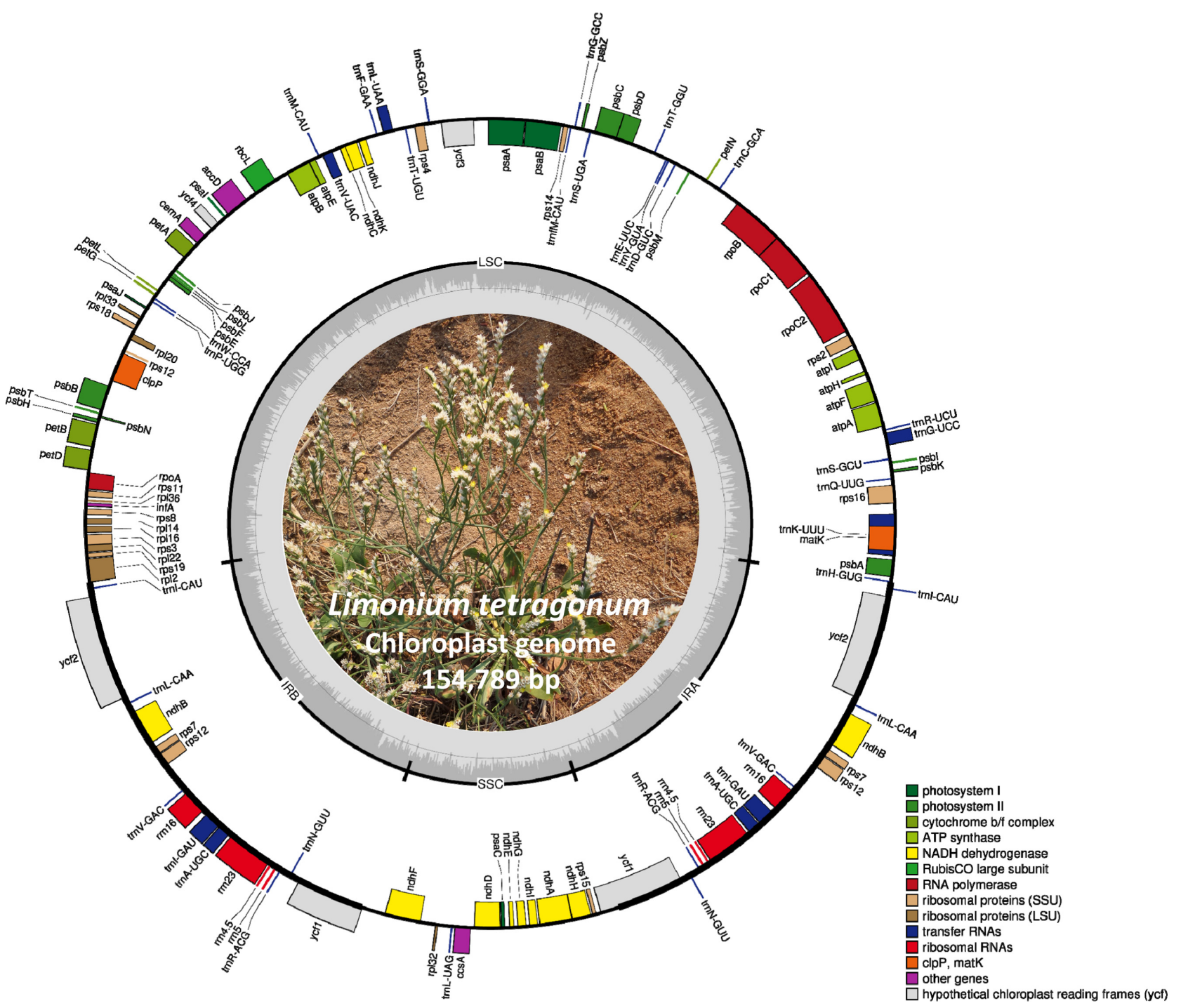

Fig. 1. Circular map of chloroplast genome of Limonium tetragonum isolated in Korea. Genes shown outside are transcribed clockwise, and those inside the circle are transcribed counter clockwise. Genes are color-coded to distinguish different functional groups. The dark grey and the light grey plot in the inner circle correspond to the GC content and AT content, respectively.

addition, seven PCGs contained at least one SNP or INDELs, among which two synonymous SNPs were found in $p s b A$ and psaA (Table 2) and four non-synonymous SNPs were identified in rpl20, rpl32, and ycf2 located in the IR region (Table 2). The ratio of synonymous to non-synonymous SNPs was 0.5 , which is different from the normal ratio, like Chenopodium album L. (Park et al., 2021a). One INDEL region was identified in the genic region of $r p o C 2$, resulting the extension of $r p o C 2$ length in comparison to the previously sequenced chloroplast genome (MW085088) (Table 2).

Sixteen chloroplast genomes were used for constructing ML and BI phylogenetic trees. Both phylogenetic trees displayed that two L. tetragonum, collected from the East and West coastal areas of Korean peninsula, respectively, were clustered with high bootstrap value (Fig. 2). The sister species of $L$. tetragonum is controversial: L. tetragonum formed a sister group with Limonium aureum (L.) Mill. in both trees (Fig. 2), whereas it was clustered with L. bicolor in the previous study using chloroplast genomes (Darshetkar et al., 2021). The close relative of $L$. tetragonum was not clearly confirmed because the phylogenetic study using chloroplast and nuclear loci could not determine the relationship with Limonium sinense (Girard) Kuntze, Limonium tenellum (Turczaninow) Kuntze, and Limonium flexuosum (Linnaeus) Kuntze (Koutroumpa et al., 2018) and the study with the complete chloroplast genome of L. tetragonum displayed that $L$. bicolor was sister species 
Table 2. List of intraspecific variations identified from the two Limonium tetragonum chloroplast genomes.

\begin{tabular}{|c|c|c|c|c|c|c|c|c|}
\hline \multirow{2}{*}{ No. } & \multirow{2}{*}{ Type } & \multicolumn{2}{|c|}{ Changed bases } & \multicolumn{2}{|c|}{ Coordination } & \multirow{2}{*}{ Type } & \multirow{2}{*}{ Position } & \multirow{2}{*}{ SNP type } \\
\hline & & MN044572 & MW085088 & MN044572 & MW085088 & & & \\
\hline 1 & SNP & G & A & 485 & 485 & Genic & psbA & Synonymous SNP \\
\hline 2 & INDEL & - & AA & 4,316 & $4,316-4,317$ & Intergenic & $\operatorname{trnK}-\mathrm{rps} 16$ & \\
\hline 3 & SNP & G & A & 4,549 & 4,551 & Intergenic & $\operatorname{trnK}-\mathrm{rps} 16$ & \\
\hline 4 & INDEL & $\mathrm{T}$ & - & 6,256 & 6,258 & Intergenic & rps16-trnQ & \\
\hline 5 & INDEL & - & AA & 6,670 & $6,671-6,672$ & Intergenic & trnQ-psbK & \\
\hline 6 & INDEL & - & $\mathrm{AAA}$ & 7,214 & $7,217-7,219$ & Intergenic & psbK-psbI & \\
\hline 7 & INDEL & - & $\mathrm{T}$ & 7,866 & 7,872 & Intergenic & $\operatorname{trnS}$-trnG & \\
\hline 8 & INDEL & - & CGGGTCA & 7,966 & 7,973-7,979 & Intergenic & $\operatorname{trnS}$-trnG & \\
\hline 9 & INDEL & - & A & 8,224 & 8,238 & Intergenic & $\operatorname{trnS}-\operatorname{trnG}$ & \\
\hline 10 & INDEL & $\mathrm{T}$ & - & 15,561 & 15,576 & Intergenic & rps2-rpoC2 & \\
\hline 11 & INDEL & - & $\mathrm{T}$ & 15,898 & 15,912 & Genic & rpoC2 & Frameshift \\
\hline 12 & SNP & $\mathrm{T}$ & G & 27,306 & 27,321 & Intergenic & rpoB-trnC & \\
\hline 13 & SNP & $\mathrm{T}$ & G & 27,307 & 27,322 & Intergenic & rpoB-trnC & \\
\hline 14 & SNP & $\mathrm{C}$ & G & 27,308 & 27,323 & Intergenic & rpoB-trnC & \\
\hline 15 & SNP & $\mathrm{C}$ & $\mathrm{A}$ & 27,309 & 27,324 & Intergenic & rpoB-trnC & \\
\hline 16 & SNP & $\mathrm{C}$ & A & 27,310 & 27,325 & Intergenic & rpoB-trnC & \\
\hline 17 & INDEL & - & A & 28,271 & 28,286 & Intergenic & trnC-petN & \\
\hline 18 & INDEL & A & - & 31,349 & 31,435 & Intergenic & $\operatorname{trnE}-\operatorname{trn} T$ & \\
\hline 19 & INDEL & - & AA & 31,472 & $31,487-31,488$ & Intergenic & $\operatorname{trnE}-\operatorname{trn} T$ & \\
\hline 20 & SNP & $\mathrm{C}$ & A & 31,768 & 31,785 & Intergenic & $\operatorname{trnE}-\operatorname{trn} T$ & \\
\hline 21 & SNP & A & G & 32,417 & 32,434 & Intergenic & $\operatorname{trnT}-p s b D$ & \\
\hline 22 & INDEL & AAAG & - & $36,846-36,849$ & 36,863 & Intergenic & psbZ-trnG & \\
\hline 23 & SNP & G & A & 42,109 & 42,122 & Genic & psaA & Synonymous SNP \\
\hline 24 & SNP & A & $\mathrm{T}$ & 42,931 & 42,944 & Intergenic & psaA-ycf3 & \\
\hline 25 & SNP & A & $\mathrm{T}$ & 42,932 & 42,945 & Intergenic & psaA-ycf3 & \\
\hline 26 & INDEL & $\begin{array}{c}\text { ATATATTTATAT } \\
\text { ATTATA }\end{array}$ & - & $47,679-47,696$ & 47,692 & Intergenic & $\operatorname{trnT}$-trnL & \\
\hline 27 & INDEL & - & TAAT & 47,873 & $47,868-47,871$ & Intergenic & $\operatorname{trn} T-\operatorname{trn} L$ & \\
\hline 28 & INDEL & ATTTAAA & - & $48,687-48,693$ & 48,686 & Intronic & $\operatorname{trnL}$ & \\
\hline 29 & SNP & A & G & 49,304 & 49,296 & Intergenic & $\operatorname{trnF}-n d h J$ & \\
\hline 30 & INDEL & - & AAAA & 55,296 & $55288-55,291$ & Intergenic & atpB-rbcL & \\
\hline 31 & INDEL & - & $\mathrm{T}$ & 55,474 & 55,470 & Intergenic & atpB-rbcL & \\
\hline 32 & INDEL & - & A & 57,897 & 57,894 & Intergenic & rbcL-accD & \\
\hline 33 & INDEL & A & - & 61,499 & 61,497 & Intergenic & ycf4-cemA & \\
\hline 34 & INDEL & - & TCTA & 63,882 & $63,879-63,882$ & Intergenic & petA-psbJ & \\
\hline 35 & SNP & G & $\mathrm{T}$ & 64,137 & 64,138 & Intergenic & petA-psbJ & \\
\hline 36 & SNP & A & $\mathrm{T}$ & 64,138 & 64,139 & Intergenic & petA-psbJ & \\
\hline 37 & SNP & A & $\mathrm{T}$ & 64,139 & 64,140 & Intergenic & petA-psbJ & \\
\hline 38 & SNP & $\mathrm{T}$ & G & 64,140 & 64,141 & Intergenic & petA-psbJ & \\
\hline
\end{tabular}


Table 2. Continued

\begin{tabular}{|c|c|c|c|c|c|c|c|c|}
\hline \multirow{2}{*}{ No. } & \multirow{2}{*}{ Type } & \multicolumn{2}{|c|}{ Changed bases } & \multicolumn{2}{|c|}{ Coordination } & \multirow{2}{*}{ Type } & \multirow{2}{*}{ Position } & \multirow{2}{*}{ SNP type } \\
\hline & & MN044572 & MW085088 & MN044572 & MW085088 & & & \\
\hline 39 & SNP & $\mathrm{G}$ & $\mathrm{T}$ & 64,141 & 64,142 & Intergenic & petA-psbJ & \\
\hline 40 & SNP & A & $\mathrm{T}$ & 64,142 & 64,143 & Intergenic & petA-psbJ & \\
\hline 41 & SNP & G & $\mathrm{T}$ & 64,143 & 64,144 & Intergenic & petA-psbJ & \\
\hline 42 & SNP & A & $\mathrm{C}$ & 64,146 & 64,147 & Intergenic & petA-psbJ & \\
\hline 43 & SNP & A & $\mathrm{T}$ & 64,147 & 64,148 & Intergenic & petA-psbJ & \\
\hline 44 & SNP & A & $\mathrm{C}$ & 64,148 & 64,149 & Intergenic & petA-psbJ & \\
\hline 45 & SNP & $\mathrm{C}$ & A & 64,149 & 64,150 & Intergenic & petA-psbJ & \\
\hline 46 & SNP & A & $\mathrm{T}$ & 64,150 & 64,151 & Intergenic & petA-psbJ & \\
\hline 47 & SNP & A & $\mathrm{T}$ & 64,151 & 64,152 & Intergenic & petA-psbJ & \\
\hline 48 & SNP & A & $\mathrm{C}$ & 64,152 & 64,153 & Intergenic & petA-psbJ & \\
\hline 49 & INDEL & $\mathrm{T}$ & - & 65,511 & 65,512 & Intergenic & psbE-petL & \\
\hline 50 & INDEL & $\mathrm{T}$ & - & 66,150 & 66,150 & Intergenic & petL-petG & \\
\hline 51 & INDEL & $\mathrm{TA}$ & - & $68,210-68,211$ & 68,209 & Intergenic & rp133-rps18 & \\
\hline 52 & INDEL & - & $\mathrm{C}$ & 68,295 & 68,292 & Intergenic & rpl33-rps18 & \\
\hline 53 & SNP & $\mathrm{T}$ & G & 69,126 & 69,124 & Genic & rpl20 & $\begin{array}{c}\text { Non-synomynous } \\
\text { SNP }\end{array}$ \\
\hline 54 & INDEL & CTTT & - & $69,359-69,362$ & 69,357 & Intergenic & rpl20-rps12 & \\
\hline 55 & SNP & $\mathrm{C}$ & A & 71,121 & 71,115 & Intronic & clpP & \\
\hline 56 & INDEL & A & - & 75,382 & 75,376 & Intergenic & psbH-petB & \\
\hline 57 & INDEL & A & - & 80,610 & 80,603 & Intergenic & rps8-rpl14 & \\
\hline 58 & INDEL & - & AAAA & 81,060 & $81,052-81,055$ & Intergenic & rpl14-rpl16 & \\
\hline 59 & SNP & $\mathrm{C}$ & A & 86,943 & 86,939 & Genic & $y c f 2$ & $\begin{array}{c}\text { Non-synomynous } \\
\text { SNP }\end{array}$ \\
\hline 60 & INDEL & - & $\mathrm{G}$ & 92,158 & 92,154 & Intergenic & ycf2-trnL & \\
\hline 61 & SNP & A & $\mathrm{T}$ & 106,466 & 106,463 & Intergenic & $\operatorname{trnR}$-trnN & \\
\hline 62 & SNP & A & $\mathrm{T}$ & 115,749 & 115,746 & Intergenic & ndhF-rpl32 & \\
\hline 63 & SNP & G & A & 116,280 & 116,277 & Genic & rpl32 & $\begin{array}{c}\text { Non-synomynous } \\
\text { SNP }\end{array}$ \\
\hline 64 & INDEL & - & $\mathrm{T}$ & 116,808 & 116,805 & Intergenic & rpl32-trnL & \\
\hline 65 & INDEL & - & TTT & 123,377 & $123,375-123,377$ & Intronic & ndhA & \\
\hline 66 & SNP & $\mathrm{T}$ & A & 132,796 & 132,797 & Intergenic & $\operatorname{trnN}-\operatorname{trn} R$ & \\
\hline 67 & INDEL & - & $\mathrm{C}$ & 147,096 & 147,097 & Intergenic & $\operatorname{trnL}-\mathrm{ycf} 2$ & \\
\hline 68 & SNP & G & $\mathrm{T}$ & 152,319 & 152,321 & Genic & $y c f 2$ & $\begin{array}{c}\text { Non-synomynous } \\
\text { SNP }\end{array}$ \\
\hline
\end{tabular}

SNP, single nucleotide polymorphism; INDEL, insertions and deletion.

(Darshetkar et al., 2021), however, number of taxa used in this study was limited due to lack of complete chloroplast genomes and L. bicolor was not included in the previous study. This incongruency of the phylogenetic relationship might be caused by different sequences in both studies. Moreover, the supportive value of the clade of L. tetragonum and L. bicolor was $73 \%$, which was lower than those $(100 \%)$ in this study (Fig. 2), suggesting that L. aureum might be a sister species of L. tetragonum. Most of Limonium phylogenetic studies were conducted with the lack of East Asian Limonium species (Lledó 


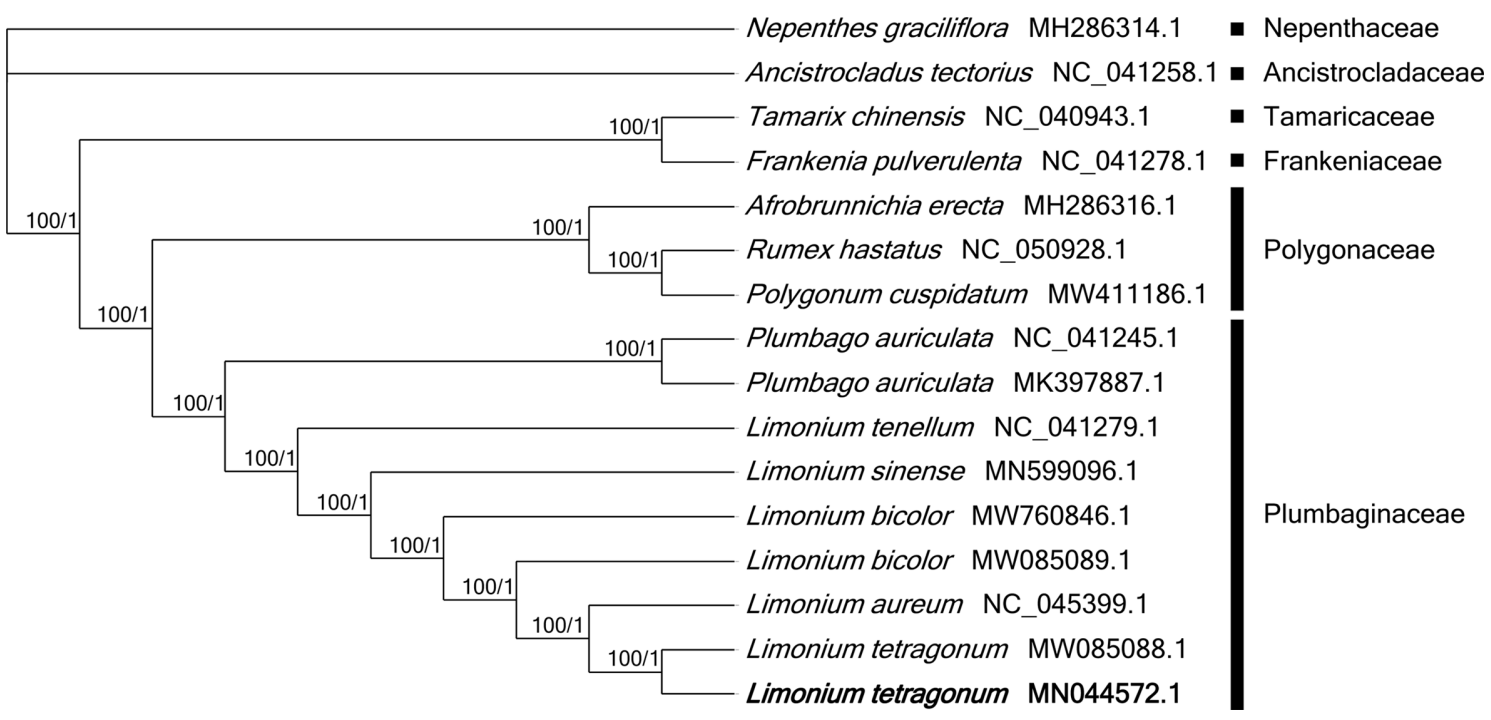

Fig. 2. Maximum-likelihood (ML) and Bayesian inference (BI) phylogenetic trees of 16 chloroplast genomes. Phylogenetic tree was drawn based on ML tree. The numbers above branches indicate support values of ML and BI trees, respectively.

et al., 2005; Malekmohammadi et al., 2017; Koutroumpa et al., 2018), requiring the additional phylogenetic studies including East Asian Limonium species.

ORCID: Yongsung KIM http://orcid.org/0000-0002-5349-9226; Jongsun PARK http://orcid.org/0000-0003-0786-4701

\section{Acknowledgments}

This study was carried out with the support of the Honam National Institute of Biological Resources (HNIBR202101107).

\section{Conflict of Interest}

The authors declare that there are no conflicts of interest.

\section{Literature Cited}

Bum, H. M., H. K Kim, N. S Kim, W. Kwon and J. Park. 2020. The complete chloroplast genome of Douinia plicata (Lindb.) Konstant. \& Vilnet (Scapaniaceae, Jungermanniales). Mitochondrial DNA Part B Resources 5: 3680-3682.

Choi, N. J., H. Xi and J. Park. 2021. A comparative analyses of the complete mitochondrial genomes of fungal endosymbionts in Sogatella furcifera, white-backed planthoppers. International Journal of Genomics 2021: 6652508.

Choi, Y. G, N. Yun, J. Park, H. Xi, J. Min, Y. Kim and S.-H Oh. 2020. The second complete chloroplast genome sequence of the Viburnum erosum (Adoxaceae) showed a low level of intra-species variations. Mitochondrial DNA Part B Resources 5: 271-272.

Crawley, S. S. and K. W. Hilu. 2012. Caryophyllales: Evaluating phylogenetic signal in trnK intron versus matK. Journal of Systematics and Evolution 50: 387-410.

Darshetkar, A. M, S. Maurya, C. Lee, B. Bazarragchaa, G. Batdelger, A. Janchiv, E. J. Jeong, S. Choi, R. K Choudhary and S.Y. Kim. 2021. Plastome analysis unveils Inverted Repeat (IR) expansion and positive selection in Sea Lavenders (Limonium, Plumbaginaceae, Limonioideae, Limonieae). PhytoKeys 175: 89-107.

Greiner, S., P. Lehwark and R. Bock. 2019. OrganellarGenomeDRAW (OGDRAW) version 1.3. 1: Expanded toolkit for the graphical visualization of organellar genomes. Nucleic Acids Research 47: W59-W64.

Huelsenbeck, J. P. and F. Ronquist. 2001. MRBAYES: Bayesian inference of phylogenetic trees. Bioinformatics 17: 754-755.

Katoh, K. and D. M. Standley. 2013. MAFFT multiple sequence alignment software version 7: Improvements in performance and usability. Molecular Biology and Evolution 30: 772-780.

Kim, M., H. Xi and J. Park. 2021. Genome-wide comparative analyses of GATA transcription factors among 19 Arabidopsis ecotype genomes: Intraspecific characteristics of GATA transcription factors. PLoS ONE 16: e0252181.

Kim, N.-H., S. H. Sung, J.-D. Heo and E. J. Jeong. 2015. The extract of Limonium tetragonum protected liver against acute alcohol toxicity by enhancing ethanol metabolism and antioxidant enzyme activities. Natural Product Sciences 21: 54-58.

Kim, N.-H., J.-D. Heo, T. B. Kim, J.-R. Rho, M. H. Yang and E. J. 
Jeong. 2016. Protective effects of ethyl acetate soluble fraction of Limonium tetragonum on diethylnitrosamine-induced liver fibrosis in rats. Biological and Pharmaceutical Bulletin 39: 1022-1028.

Kim, Y., K.-I. Heo, S. Lee, and J. Park. 2018. Complete chloroplast genome sequence of the Pseudostellaria longipedicellata S. Lee, K. Heo \& S.C. Kim (Caryophyllaceae). Mitochondrial DNA Part B Resources 3: 1296-1297.

Kim, Y., Y Chung and J. Park. 2019a. The complete chloroplast genome sequence of Dysphania pumilio (R. Br.) Mosyakin \& Clemants (Amaranthaceae). Mitochondrial DNA Part B Resources 4: 403-404.

Kim, Y., Y. J. Oh, K. Y. Han, G. H. Kim, J. Ko and J. Park. 2019b. The complete chloroplast genome sequence of Hibiscus syriacus L. 'Mamonde' (Malvaceae). Mitochondrial DNA Part B Resources 4: 558-559.

Kim, Y, J. Park and Y. Chung. 2019c. Comparative analysis of chloroplast genome of Dysphania ambrosioides (L.) Mosyakin \& Clemants understanding phylogenetic relationship in genus Dysphania R. Br. Korean Journal of Plant Resources 32: 644-668.

Kim, Y., J.-S. Yi, J. Min, H. Xi, D. Y. Kim, J. Son, J. Park and J.-I. Jeon. 2019d. The complete chloroplast genome of Aconitum coreanum (H. Lév.) Rapaics (Ranunculaceae). Mitochondrial DNA Part B Resources 4: 3404-3406.

Kong, C.-S., Y. R. Um, J.-I. Lee, Y. A. Kim, J.-S. Lee and Y. Seo. 2008. Inhibition effects of extracts and its solvent fractions isolated from Limonium tetragonum on growth of human cancer cells. Korean Journal of Biotechnology and Bioengineering 23: 177-182.

Koutroumpa, K., S. Theodoridis, B. H. Warren, A. Jiménez, F. Celep, M. Doğan, M. M. Romeiras, A. Santos-Guerra, J. M. Fernández-Palacios, J. Caujapé-Castells, M. Moura, M. M. de Sequeira and E. Conti. 2018. An expanded molecular phylogeny of Plumbaginaceae, with emphasis on Limonium (sea lavenders): Taxonomic implications and biogeographic considerations. Ecology and Evolution 8: 12397-12424.

Kubitzki, K. 1993. Plumbaginaceae. In The Families and Genera of Vascular Plants. Vol. 2. Kubitzki, K., J. Rohwer and V. Bittrich (eds.), Springer-Verlag, Berlin. Pp. 523-530.

Lee, J. I., C.-S. Kong, M. E. Jung, J. W. Hong, S. Y. Lim and Y. Seo. 2011. Antioxidant activity of the halophyte Limonium tetragonum and its major active components. Biotechnology and Bioprocess Engineering 16: 992.

Lee, Y.-E, Y. Lee Y and S. Kim. 2021. A report of the second chloroplast genome sequence in Veronica nakaiana (Plantaginaceae), an endemic species in Korea. Korean Journal of Plant Taxonomy 51: 109-114.
Li, H. 2013. Aligning sequence reads, clone sequences and assembly contigs with BWA-MEM. arXiv preprint at https:// arxiv.org/abs/1303.3997.

Li, H., B. Handsaker, A. Wysoker, T. Fennell, J. Ruan, N. Homer, G. Marth, G. Abecasis and R. Durbin. 2009. The sequence alignment/map format and SAMtools. Bioinformatics 25: 2078-2079.

Lledó, M. D., M. B. Crespo, M. F. Fay and M. W. Chase. 2005. Molecular phylogenetics of Limonium and related genera (Plumbaginaceae): Biogeographical and systematic implications. American Journal of Botany 92: 1189-1198.

Malekmohammadi, M., H. Akhani and T. Borsch. 2017. Phylogenetic relationships of Limonium (Plumbaginaceae) inferred from multiple chloroplast and nuclear loci. Taxon 66: 11281146.

Min, J., W. Kwon, H. Xi and J. Park. 2019a. The complete chloroplast genome of Leucobryum juniperoideum (brid.) C. Müll. (Leucobryaceae, Bryophyta). Mitochondrial DNA Part B Resources 4: 2962-2963.

Min, J., J. Park, Y. Kim and W. Kwon. 2019b. The complete chloroplast genome of Artemisia fukudo Makino (Asteraceae): Providing insight of intraspecies variations. Mitochondrial DNA Part B Resources 4: 1510-1512.

Morgan, E., and K, Funnell. 2018. Limonium. In Ornamental Crops. Van Huylenbroeck, J. (ed.), Springer, Cham. Pp. 513527.

Nguyen, L.-T., H. A. Schmidt, A. Von Haeseler and B. Q. Minh. 2015. IQ-TREE: A fast and effective stochastic algorithm for estimating maximum-likelihood phylogenies. Molecular Biology and Evolution 32: 268-274.

Ohwi, J. 1965. Flora of Japan (rev. ed.). Shibundo Co. Ltd., Tokyo, $344 \mathrm{pp}$.

Park, C.-W.. 2007. The Genera of Vascular Plants of Korea. Flora of Korea Editorial Committee. Academy Publishing Co, Seoul, $1482 \mathrm{pp}$.

Park, J. and S.-H Oh. 2020. A second complete chloroplast genome sequence of Fagus multinervis Nakai (Fagaceae): Intraspecific variations on chloroplast genome. Mitochondrial DNA Part B Resources 5: 1868-1869.

Park, J., J.-H. An, Y. Kim, D. Kim, B.-G. Yang and T. Kim. 2020a. Database of National Species List of Korea: The taxonomical systematics platform for managing scientific names of Korean native species. Journal of Species Research 9: 233-246.

Park, J., J. Min, Y. Kim and Y. Chung. 2021a. The comparative analyses of six complete chloroplast genomes of morphologically diverse Chenopodium album L. (Amaranthaceae) collected in Korea. International Journal of Genomics 2021: 6643444. 
Park, J., S. Park, T. Jang, G. Kim and J.-H. Park. 2021b. The complete chloroplast genome of Abeliophyllum distichum f. lilacinum Nakai (Oleaceae) from the Chungbuk Province, Korea. Mitochondrial DNA Part B Resources 6: 1754-1756.

Park, J., H. Xi, J. Son, H.T. Shin, H. Kang and S. Park. 2021c. The complete chloroplast genome of Castanopsis sieboldii (Makino) Hatus (Fagaceae). Mitochondrial DNA Part B Resources 6: 2743-2745.

Park, J., H. Xi and Y. Kim. 2021d. The mitochondrial genome of Arabidopsis thaliana (Brassicaceae) isolated in Korea. Korean Journal of Plant Taxonomy. 51: 176-180.

Yang, M. H., N.-H. Kim, J.-D. Heo, S. H. Sung and E. J. Jeong. 2014. Hepatoprotective effects of Limonium tetragonum, edible medicinal halophyte growing near seashores. Pharmacog- nosy Magazine 10: S563-S568.

Yao, G., J.-J. Jin, H.-T. Li, J.-B. Yang, V. S. Mandala, M. Croley, R. Mostow, N. A. Douglas, M. W. Chase, M. J. M. Christenhusz, D. E. Soltis, P. S. Soltis, S. A. Smith, S. F. Brockington, M. J. Moore, T.-S. Yi and D.-Z. Li. 2019. Plastid phylogenomic insights into the evolution of Caryophyllales. Molecular Phylogenetics and Evolution 134: 74-86.

Zerbino, D. R. and E. Birney. 2008. Velvet: Algorithms for de novo short read assembly using de Bruijn graphs. Genome Research 18: 821-829.

Zhao, Q.-Y., Y. Wang Y, Y.-M. Kong, D. Luo, X. Li and P. Hao. 2011. Optimizing de novo transcriptome assembly from shortread RNA-Seq data: A comparative study. BMC Bioinformatics 12: S2. 